\title{
Endoscopic ultrasound-guided hemostasis of rectal varices
}

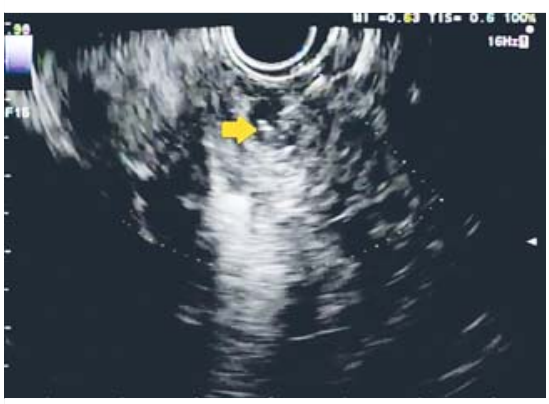

- Fig. 1 Endoscopic ultrasound showing deployment of embolization coils (yellow arrow).

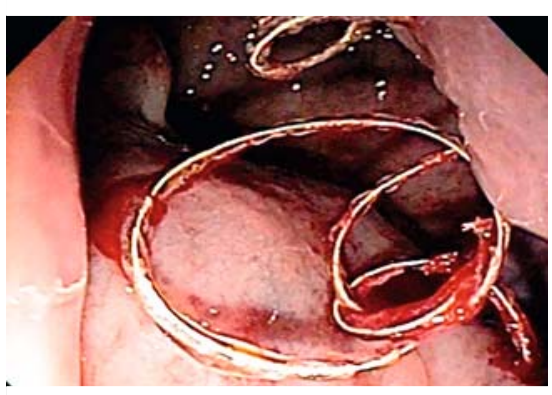

- Fig. 2 Endoscopy showing the embolization coils anchored in the rectal mucosa.

The prevalence of rectal varices in patients with cirrhosis ranges from $38 \%$ to $56 \%$ [1]. While lower gastrointestinal endoscopy can help diagnose rectal varices, endoscopic ultrasound (EUS) may be more accurate in patients with smaller varices [1]. Reports are limited, but techniques for EUS-guided hemostasis of rectal varices include: injection sclerotherapy [2], band ligation [3], embolization with coils [4], and glue injection [4,5]. Interventional radiology procedures, such as transjugular intrahepatic portosystemic shunting and balloon-occluded retrograde transvenous obliteration, are helpful only in selected patients. Here we describe a unique case of EUS-guided hemostasis of rectal varices using coils and glue.

Our patient was a 54-year-old man with a history of chronic hepatitis C (genotype $1 \mathrm{~b}$, treatment-naïve) who presented with a 1-month history of hematochezia. His vital signs were blood pressure of $120 / 70 \mathrm{mmHg}$ and heart rate of 70 beats per minute. Initial laboratory tests revealed the following results: hemoglobin $9.1 \mathrm{~g} / \mathrm{dL}$, platelets $67000 / \mu \mathrm{L}$, and he had a MELD score of 11.

Colonoscopy revealed large rectal varices. On EUS, a grape-like bunch of rectal varices was seen, which showed sluggish blood flow on Doppler exam. It was decided to treat the varices with embolization coils and glue. A 22-gauge EUSguided fine needle aspiration (FNA) needle (EchoTip Ultra; Cook Medical, Limerick, Ireland) and embolization coils (MicroNester; Cook Medical, Bjaeverskov, Denmark) were used.

The EUS-FNA needle was used to puncture the feeder vessel. One $10-\mathrm{mm} \times$ $7-\mathrm{cm}$ coil was anchored into the wall of the feeder vessel and deployed into the lumen under sonographic guidance ( $\triangleright$ Fig. 1). Another 10-mm × 7-cm embolization coil was similarly deployed in an adjacent feeder vessel. A further medium varix was identified, and an $8-\mathrm{mm} \times$ 14-cm embolization coil was deployed, giving a total of three coils deployed in two columns. Endoscopy showed the proximal end of the coil anchored in the rectal mucosa ( $\triangleright$ Fig.2). Under di- rect endoscopic view with EUS assistance, $0.8 \mathrm{~mL}$ of n-butyl-2-cyanoacrylate glue (Covidien SwiftSet; United Kingdom) was injected into the rectal varix at the site of coil deployment. Doppler examination confirmed a reduction in blood flow after coil placement and glue injection ( $\triangleright$ Video 1 ).

At 4-week follow-up, our patient reported no further rectal bleeding and his hemoglobin was stable. There were no procedural complications.

Endoscopy_UCTN_Code_TTT_1AQ_2AZ

Competing interests

None

The Authors

Tanima Jana*, Tejal Mistry*, Shashideep Singhal

Department of Internal Medicine, Division of Gastroenterology, Hepatology \& Nutrition, Houston Health Sciences Center, Houston, Texas, USA

* Joint first authors

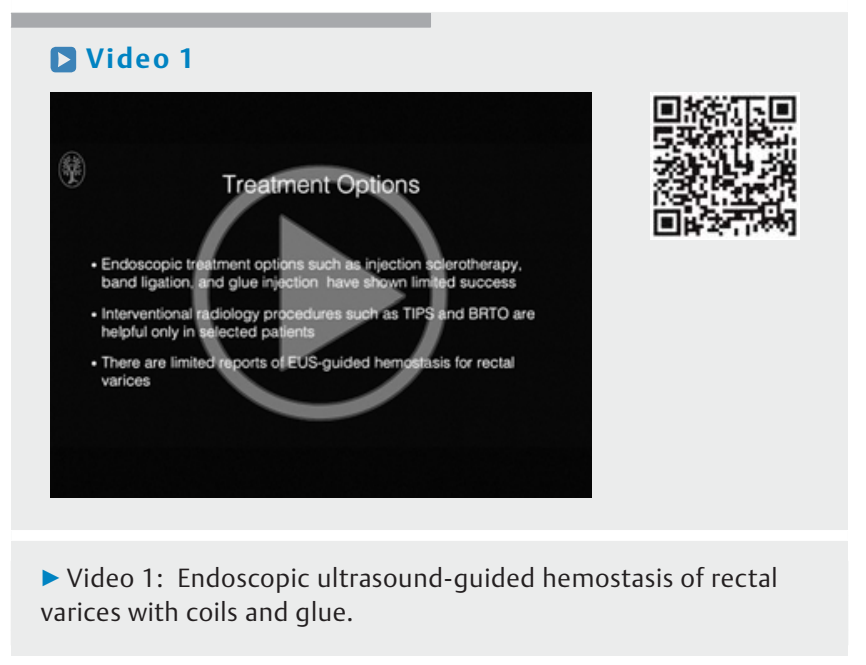




\section{Shashideep Singhal, MD}

Department of Internal Medicine, Division of Gastroenterology, Hepatology \& Nutrition, University of Houston Health Sciences Center, Houston, TX 77030, USA

Fax: +1-713-500-6699

Phone: +1-713-500-6631

sdsinghal@gmail.com

\section{References}

[1] Al Khalloufi K, Laiyemo AO. Management of rectal varices in portal hypertension. World ] Hepatol 2015; 7: $2992-2998$

[2] Connor EK, Duran-Castro OL, Attam R. Therapy for recurrent bleeding from rectal varices by EUS-guided sclerosis. Gastrointest Endosc 2015; 81: 1280 - 1281

[3] Sharma M, Rai P, Bansal R. EUS-assisted evaluation of rectal varices before banding. Gastroenterol Res Pract 2013; 2013: 619187

[4] Weilert F, Shah JN, Marson FP et al. EUSguided coil and glue for bleeding rectal varix. Gastrointest Endosc 2012; 76: 915-916

[5] Sharma M, Somasundaram A. Massive lower Gl bleed from an endoscopically inevident rectal varices: diagnosis and management by EUS (with videos). Gastrointest Endosc 2010; 72: $1106-1108$

\section{Bibliography}

DOI http://dx.doi.org/10.1055/s-0043-104521 Endoscopy 2017; 49: E136-E137

(c) Georg Thieme Verlag KG

Stuttgart · New York

ISSN 0013-726X 